\title{
ESTRESSE OCUPACIONAL ENTRE PROFISSIONAIS DE ENFERMAGEM DO BLOCO CIRÚRGICO
}

\author{
Denise Rodrigues Costa Schmidt ${ }^{1}$, Rosana Aparecida Spadoti Dantas ${ }^{2}$, Maria Helena Palucci Marziale ${ }^{3}$, \\ Ana Maria Laus4
}

\footnotetext{
${ }^{1}$ Doutoranda do Programa de Doutorado em Enfermagem Fundamental da Escola de Enfermagem de Ribeirão Preto (EERP) da Universidade de São Paulo (USP). Enfermeira do Hospital Universitário de Londrina. Paraná, Brasil. E-mail: denisebeto@ terra.com.br

${ }^{2}$ Doutora em Enfermagem. Professor Associado do Departamento de Enfermagem Geral e Especializada da EERP/USP. São Paulo, Brasil. E-mail: rsdantas@eerp.usp.br

${ }^{3}$ Doutora em Enfermagem. Professor Titular do Departamento de Enfermagem Geral e Especializada da EERP/USP. São Paulo, Brasil. E-mail: marziale@eerp.usp.br

${ }^{4}$ Doutora em Enfermagem. Professor Doutor do Departamento de Enfermagem Geral e Especializada da EERP/USP. São Paulo, Brasil. E-mail: analaus@eerp.usp.br
}

\begin{abstract}
RESUMO: Estudo descritivo e correlacional, de corte transversal cujos objetivos foram avaliar a presença de estresse ocupacional entre os profissionais de enfermagem do bloco cirúrgico e possíveis associações entre o estresse ocupacional e as características profissionais. O modelo Demanda-Controle de Karasek foi utilizado para essa avaliação. A amostra foi constituída por 211 trabalhadores de enfermagem de 11 hospitais da cidade de Londrina-PR, os quais responderam a Job Stress Scale. A coleta de dados ocorreu no período de abril a novembro de 2007. A maioria dos participantes era de auxiliares de enfermagem (62,6\%), do sexo feminino $(86,7 \%)$, casados (54,0\%), com idade média de 40 anos e com exposição intermediária ao estresse ocupacional (56,1\%). As dimensões Demanda, Controle e Apoio Social obtiveram média de 14,8 (DP=2,4), $16,5(\mathrm{DP}=2,3)$ e 18,7 (DP=2,8), respectivamente. Demanda obteve associação estatisticamente significante com a categoria profissional $(\mathrm{p}=0,01)$, enquanto Controle apresentou-se estatisticamente significante com o tipo de instituição, categoria profissional e carga horária semanal $(\mathrm{p}<0,05)$.
\end{abstract}

DESCRITORES: Estresse. Enfermagem. Centros de cirurgia.

\section{OCCUPATIONAL STRESS AMONG NURSING STAFF IN SURGICAL SETTINGS}

\begin{abstract}
This descriptive, correlation, and cross-sectional study aimed to evaluate the presence of occupational stress among nursing professionals working in surgical settings and to investigate relations between occupational stress and the work characteristics. The Demand-Control Model proposed by Karasek was used to evaluate occupational stress. The sample was composed of 211 nursing professionals from 11 hospitals located in the city of Londrina-PR, Brazil. A questionnaire regarding socio-demographic and professional data and the Job Stress Scale were applied. Data was collected from April to November, 2007. Among the participants, most were auxiliary nurses $(62.6 \%)$, women $(86.7 \%)$, and married $(54.0 \%)$. The average age was 40 years. The average for Demand, Control, and Social Support dimensions was 14.8 (S.D.=2.4), $16.5($ S.D. $=2.3)$ and 18.7 (S.D.=2.8), respectively. Considering the results related to the Job Stress Scale, the Demand dimension showed a statistically significant association with the professional category ( $p=0.01$ ), and Control showed a statistically significant association with the type of hospital, professional category, and weekly working hours $(\mathrm{p}<0.05)$.
\end{abstract}

DESCRIPTORS: Stress. Nursing. Surgicenters.

\section{ESTRÉS OCUPACIONAL ENTRE PROFESIONALES DE ENFERMERÍA QUE LABORAN EN UNIDADES QUIRÚRGICAS}

RESUMEN: Se trata de un estudio de correlación, descriptivo y transversal, cuyo objetivo fue evaluar la incidencia de estrés laboral de trabajadores de enfermería que laboran en unidades quirúrgicas y las posibles asociaciones entre la medida de estrés laboral y las características del trabajo. El modelo Demanda-Control de Karasek fue utilizado para evaluar el estrés laboral. La muestra fue constituida por 211 profesionales de enfermería de 11 hospitales de la ciudad de Londrina-PR, Brasil. Los participantes respondieron a un cuestionario de datos generales, ocupacionales y la Job Stress Scale. La recolección de los datos fue hecha en el período de abril a noviembre de 2007. Los resultados demostraron que la mayoría eran auxiliares de enfermería (62,6\%), del sexo femenino (86,7\%), casados $(54,0 \%)$, con media de edad de 40 años y con exposición intermediaria al estrés ocupacional (56,1\%). Las medias relacionadas a las dimensiones Demanda, Control y Apoyo Social fueron de 14,8 (D.P.=2,4), 16,5 (D.P.=2,3) y 18,7 (D.P.=2,8), respectivamente. Las asociaciones cuyo resultado fue estadísticamente significante ocurrieron entre la Demanda y la categoría profesional ( $\mathrm{p}=0,01)$, el Control y tipos de hospitales, categoría profesional y carga horaria semanal $(p<0,05)$.

DESCRIPTORES: Estrés. Enfermería. Centros quirúrgicos. 


\section{INTRODUÇÃO}

O estresse ocupacional no modo de vida atual tornou-se uma importante fonte de preocupação e é reconhecido como um dos riscos mais sérios ao bem-estar psicossocial do indivíduo. Várias pesquisas descrevem a complexidade do tema e a necessidade de outros estudos sobre a etiologia do problema. $\mathrm{O}$ estresse relacionado ao trabalho coloca em risco a saúde dos membros da organização e tem como consequências o desempenho ruim, baixo moral, alta rotatividade, absenteísmo e violência no local de trabalho. ${ }^{1}$

O estresse contínuo relacionado ao trabalho também constitui um importante fator determinante dos transtornos depressivos e de outras doenças, tais como, síndrome metabólica, síndrome da fadiga crônica, distúrbios do sono, diabetes e a síndrome de Burnout. ${ }^{2-3}$

Avaliar a presença de estresse no trabalho, entretanto, não é uma tarefa fácil. A complexidade do fenômeno tem levado à formulação de uma multiplicidade de conceitos para o termo e uma variedade de modelos de análise que ainda demonstram fragilidade de várias ordens. ${ }^{2}$ Quanto ao conceito, a Organização Internacional do Trabalho define o estresse ocupacional como sendo um conjunto de fenômenos que se apresentam no organismo do trabalhador e que, por esse motivo, pode afetar sua saúde. Os principais fatores geradores de estresse presentes no ambiente de trabalho envolvem os aspectos da organização, administração e sistema de trabalho e da qualidade das relações humanas. ${ }^{4}$

Dentre as várias abordagens utilizadas em estudos sobre estresse no ambiente de trabalho optamos pelo uso do modelo Demanda - Controle que não se preocupa em medir fatores de personalidade ou estressores externos ao trabalho e considera os fatores individuais, como estilos de enfrentamento ao estresse, como modificadores do processo. ${ }^{5}$ Para os autores desse modelo, alguns modelos de análise introduzem a dimensão individual de forma precoce, deixando de avaliar os fatores ambientais determinantes. ${ }^{6}$

O modelo Demanda - Controle é um modelo bidimensional que relaciona os níveis de controle do trabalhador sobre o próprio trabalho e de demandas psicológicas oriundas do ambiente laboral e as repercussões sobre a estrutura psíquica e orgânica dos trabalhadores. O modelo prevê que o estresse no trabalho é resultante da interação entre muitas demandas psicológicas, menor controle no processo de trabalho e menor apoio social recebido de colaboradores e chefes no ambiente laboral. ${ }^{5}$
Com base na avaliação simultânea de níveis de demanda e de controle, quatro experiências poderiam acontecer no ambiente de trabalho: trabalho com alta exigência (alta demanda e baixo controle); trabalho ativo (alta demanda e alto controle); trabalho de baixa exigência (baixa demanda e alto controle) e trabalho passivo (baixa demanda e baixo controle). ${ }^{5}$

Concernente à enfermagem do bloco cirúrgico, o estresse está presente no seu cotidiano, resultante de inúmeros fatores relacionados ao tipo de ambiente, complexidade das relações humanas e de trabalho, autonomia profissional, grau elevado de exigência quanto às competências e habilidades, alta responsabilidade, planejamento adequado de recursos humanos e materiais, entre outros, ${ }^{7-9}$ o que aponta para a grande importância de realização de estudos direcionados a esse grupo de trabalhadores. Ao se considerar esses aspectos, desenvolvemos o presente estudo que teve por objetivos avaliar a presença de estresse ocupacional entre os profissionais de enfermagem que atuam nos blocos cirúrgicos do município de Londrina, e analisar as possíveis associações entre a medida de estresse ocupacional e o tipo de instituição hospitalar, a categoria profissional, a carga horária semanal e a atuação em mais de uma instituição de saúde.

\section{MÉTODO}

Estudo descritivo e correlacional, de corte transversal, desenvolvido nos blocos cirúrgicos de 11 hospitais da cidade de Londrina - PR e aprovado pelo Comitê de Bioética e Ética em Pesquisa da Irmandade Santa Casa de Londrina, sob o $\mathrm{N}^{\circ}$ 235/06, de acordo com as orientações da Resolução No 196/96 do Conselho Nacional de Saúde.

A população do estudo foi composta pelos profissionais da equipe de Enfermagem do Centro Cirúrgico (CC) e/ou Central de Materiais e Esterilização (CME) de 16 hospitais da cidade de Londrina, dos quais apenas 11 permitiram a coleta de dados, totalizando 340 trabalhadores nos Blocos Cirúrgicos (BCs) no momento da coleta de dados, para os quais foram entregues os instrumentos da pesquisa.

A amostra foi composta por $211(66,8 \%)$ trabalhadores de enfermagem (enfermeiros, técnicos, auxiliares e atendentes de enfermagem) que concordaram em participar do estudo, assinando o Termo de Consentimento Livre e Esclarecido e retornando o instrumento de coleta de dados preenchido para as pesquisadoras. 
A coleta de dados foi realizada por meio dos questionários entregues em mãos aos potenciais participantes, nos meses de maio a novembro de 2007 , sendo que aqueles que foram preenchidos retornaram às pesquisadoras no prazo estabelecido de dez dias após a entrega, em envelopes fechados que permitiram o anonimato dos respondentes.

O instrumento de coleta dos dados era composto por duas partes, a primeira relacionada à caracterização dos trabalhadores, contendo dados sócio-demográficos (idade, sexo, situação conjugal e escolaridade) e profissionais (categoria profissional, instituição de origem, carga horária de trabalho semanal, presença de outro vínculo empregatício, remuneração recebida, segundo o salário-mínimo vigente no período da coleta dos dados) e a segunda contendo a versão para o português da Job Stress Scale (JSS), ${ }^{10}$ elaborada originalmente na Suécia para a avaliação do estresse ocupacional. ${ }^{11}$

Com o objetivo de identificar possíveis dificuldades no preenchimento do instrumento pelos potenciais participantes, realizamos um pré-teste em abril de 2007. Participaram do mesmo 20 trabalhadores de enfermagem escolhidos aleatoriamente, por meio da escala de revezamento, dos setores envolvidos na pesquisa e que atuavam em dois hospitais que participaram do estudo. O retorno obtido foi de 15 questionários preenchidos e a análise destes permitiu identificar que a JSS não necessitaria de mudanças em sua redação ou formato.

A JSS contém 17 itens que são distribuídos da seguinte forma na escala: cinco itens avaliam demanda, seis avaliam controle e seis analisam apoio social. Dentre os itens que avaliam a dimensão demanda, quatro se referem a aspectos quantitativos como tempo e velocidade para a realização do trabalho (Com que frequência você tem que fazer suas tarefas de trabalho com muita rapidez? Com que frequência você tem que trabalhar intensamente, isto é, produzir muito em pouco tempo? Seu trabalho exige demais de você? Você tem tempo suficiente para cumprir todas as tarefas de seu trabalho?) e uma questão analisa aspectos predominantemente qualitativos do processo de trabalho relacionados ao conflito entre diferentes demandas $(\mathrm{O}$ seu trabalho costuma apresentar exigências contraditórias ou discordantes?). Dentre os seis itens da dimensão controle, quatro avaliam o uso de desenvolvimento de habilidades (Você tem possibilidade de aprender coisas novas em seu trabalho? Seu trabalho exige muita habilidade ou conhecimentos especializados? Seu trabalho exige que você tome iniciativas? No seu trabalho, você tem que repetir muitas vezes as mesmas tarefas?) e dois estão relacionados à autonomia para tomada de decisão sobre o processo de trabalho (itens Você pode escolher como fazer o seu trabalho? Você pode escolher o que fazer no seu trabalho?). A dimensão apoio social possui seis itens (Existe um ambiente calmo e agradável onde trabalho; no trabalho, nos relacionamos bem uns com os outros; eu posso contar com o apoio de meus colegas de trabalho; se eu não estiver num bom dia, meus colegas compreendem; no trabalho, eu me relaciono bem com meus chefes; e eu gosto de trabalhar com meus colegas.) os quais avaliam o apoio que o trabalhador recebe da chefia e dos colegas de trabalho. ${ }^{10}$

Trata-se de uma escala do tipo Likert de quatro pontos, variando de frequentemente (4) a nunca (1) para a escala de demanda e controle, e de concordo totalmente (4) a discordo totalmente (1) para a escala de apoio social. ${ }^{10}$

Alguns itens das dimensões demanda e controle possuem escores reversos e devem ser examinados antes do cálculo da dimensão a qual pertencem. O procedimento de cálculo do escore da dimensão demanda é dado pela soma dos escores atribuídos aos cinco itens (após a inversão do escore de um dos seus itens) que compõem a dimensão e pode variar de cinco a 20, quanto maior o escore maior a demanda psicológica percebida pelo trabalhador. Na dimensão controle há outro item com valor reverso que precisa ser recodificado. Essa dimensão pode ser dividida em duas subescalas: "discernimento intelectual" (quatro itens) e "autoridade sobre decisões" (dois itens). Em nosso estudo optamos por trabalhar apenas com o escore total da dimensão controle, cujo resultado é obtido pela soma dos escores das duas subescalas e varia de 6 a 24, o que significa que quanto maior o valor, maior o controle de como e quando o profissional desenvolve seu trabalho. $\mathrm{O}$ escore da dimensão apoio social possui intervalo de 6 a 24 e é dado pela soma das respostas fornecidas aos seis itens, o que significa que quanto maior o escore, melhor o apoio social do trabalhador em seu ambiente de trabalho. ${ }^{10}$

Neste estudo, optamos primeiramente por utilizar os escores contínuos para as três dimensões que compõem a JSS, pois julgamos que essa análise seria mais eficaz para obter uma compreensão inicial dos dados coletados e, em seguida, utilizamos a classificação proposta no estudo de outros autores, ${ }^{12}$ os quais sugerem que trabalhadores expostos a uma combinação de alta demanda e baixo controle (alta exigência) são considerados 
como grupo de maior exposição ao estresse ocupacional; aqueles expostos à alta demanda, mas tendo alto controle (trabalho ativo) ou a baixo controle e baixa demanda (trabalho passivo) são considerados como grupo de exposição intermediária ao estresse ocupacional e aqueles com alto controle e baixa demanda (baixa exigência) são classificados como não expostos ao estresse no trabalho. Para identificação dos grupos de alta e baixa demanda e do grupo de alto e baixo controle utilizamos as medianas das referidas dimensões, formando duas categorias para a dimensão demanda e duas para controle.

Os dados coletados foram processados e analisados no programa Statistical Package for the Social Science (SPSS) version 15.0 for Windows. Foram realizadas análises descritivas para todas as variáveis. O teste de Kolmogorov-Smirnov foi realizado para verificar a normalidade da distribuição das medidas obtidas pela JSS. Para verificarmos possíveis associações do estresse ocupacional com as variáveis: tipo de instituição hospitalar (pública/ filantrópica ou privada); atuação em mais de uma instituição de saúde (sim ou não), carga horária semanal (até 40 horas ou acima de 40 horas semanais) e categoria profissional (enfermeiro ou não enfermeiro*) utilizamos teste $\mathrm{t}$ de Student e coeficiente de correlação de Pearson (quando confirmamos a normalidade da distribuição da medida) ou teste Mann Whitney e de correlação de Spearman (quando a normalidade não foi confirmada). O nível de significância adotado foi de 0,05.

Com relação ao tratamento dos dados perdidos (missing data), ou seja, quando os participantes deixaram de responder itens da escala, seguimos o critério que estabelece que só deva ser excluídos da amostra aqueles participantes que tiveram $20 \%$ ou mais de itens não respondidos. ${ }^{13}$ Diante desse critério foram mantidos para análise dessas medidas os participantes que tivessem respondido, no mínimo, quatro itens da dimensão demanda e cinco itens das dimensões controle e apoio social da JSS versão resumida. As consistências internas das dimensões da JSS foram analisadas pelo Alfa de Cronbach e o valor adotado como critério de boa consistência interna foi de $0,70 .{ }^{14}$

\section{RESULTADOS}

Os participantes do estudo eram predominantemente do sexo feminino $(86,7 \%)$ e com idade variando de 20 a 68 anos, com mediana e média de 40 e Desvio-Padrão (DP) de 9,7 anos. Quanto à situação conjugal $114(54,0 \%)$ eram casados, $51(24,2 \%)$ solteiros, $35(16,6 \%)$ separados e nove $(4,3 \%)$ viúvos. A escolaridade variou entre os trabalhadores: $21(9,9 \%)$ com o ensino fundamental completo, $123(58,3 \%)$ com ensino médio completo, 31 (14,7\%) com curso superior incompleto e $36(17,1 \%)$ com curso superior completo, sendo que desses, 16 (7,6\% do total) possuíam especialização, mestrado ou doutorado.

Considerando as categorias profissionais, a de maior frequência foi a dos auxiliares (132; $62,6 \%)$, seguida pelas categorias de atendente (28; $13,3 \%)$, técnico de enfermagem $(27 ; 12,8 \%)$ e enfermeiro (22; 10,4\%).

No que se refere aos tipos de instituição hospitalar nas quais os participantes atuavam, cinco eram públicas ou filantrópicas e seis privadas, sendo que, $123(58,3 \%)$ trabalhadores atuavam em instituições públicas/filantrópicas e 88 (41,7\%) em hospitais privados, com tempo médio de experiência nos $\mathrm{BCs}$ dessas instituições de 9,3 (DP=8) anos.

Com relação aos dados perdidos da JSS um participante foi excluído da análise dos três componentes da escala; três foram excluídos da análise do domínio apoio social, um da análise do domínio demanda e um do domínio controle, enquanto cinco participantes tiveram seus dados perdidos substituídos pela média dos demais itens do domínio.

Os resultados relacionados às variáveis de interesse, as medidas das dimensões demanda e controle obtidas, por meio da aplicação da JSS, estão apresentados na Tabela 1.

Tabela 1 - Estatística descritiva e consistência interna das dimensões demanda, controle e apoio social dos trabalhadores de enfermagem. Londrina-PR, 2007

\begin{tabular}{lcccc}
\hline $\begin{array}{l}\text { Dimensões } \\
\text { da Job } \\
\text { stress scale }\end{array}$ & $\begin{array}{c}\text { Alfa de } \\
\text { Cronbach }\end{array}$ & $\begin{array}{c}\text { Média } \\
\text { (DP*) }\end{array}$ & Mediana & $\begin{array}{c}\text { Intervalo } \\
\text { possível }\end{array}$ \\
\hline Demanda & 0,73 & $14,8(2,4)$ & 15,0 & $5-20$ \\
Controle & 0,54 & $16,5(2,3)$ & 17,0 & $6-24$ \\
Apoio Social & 0,75 & $18,7(2,8)$ & 19,0 & $6-24$ \\
\hline * Desvio Padrão & & & &
\end{tabular}

Ao analisarmos o escore geral para as dimensões da JSS e seu respectivo intervalo possível, observamos que houve escores elevados para as

\footnotetext{
* Os profissionais das categorias técnico, auxiliar e atendente de enfermagem foram denominados, neste estudo, de profissionais não enfermeiros.
} 
três sub-dimensões, podendo indicar um grupo de risco para o estresse ocupacional, apesar do grupo de trabalhadores perceber alto apoio social no ambiente de trabalho (mediana $=19,0$ ). Quanto à avaliação da consistência interna do instrumento, avaliada pelo Alfa de Cronbach, observamos que somente a dimensão Controle obteve valor baixo $(0,54)$ quando comparada às demais dimensões.

Ao utilizarmos o critério para a classificação dos trabalhadores segundo a exposição ao estres- se ocupacional, ${ }^{12}$ observamos que a maioria dos trabalhadores de enfermagem se encontrava na categoria de exposição intermediária ao estresse ocupacional (56,1\%), 54 (26,3\%) encontravam-se na categoria de alta exposição ao estresse no trabalho, enquanto apenas $36(17,6 \%)$ consideravam-se sem exposição ao estresse.

Na Tabela 2 apresentamos a associação das dimensões da JSS com as variáveis profissionais dos trabalhadores de enfermagem dos BCs.

Tabela 2 - Estatística descritiva da dimensão demanda segundo a associação com as variáveis profissionais dos trabalhadores de enfermagem. Londrina-PR, 2007

\begin{tabular}{|c|c|c|c|c|c|}
\hline \multirow[t]{2}{*}{ Variáveis } & \multicolumn{5}{|c|}{ Demanda } \\
\hline & $\mathrm{n}$ & Amplitude & Mediana & Média $\left(\mathrm{DP}^{*}\right)$ & $\mathrm{p} \dagger$ \\
\hline \multicolumn{6}{|l|}{ Tipo de instituição } \\
\hline Pública/filantrópica & 111 & $6-19$ & 15,0 & $14,6(2,4)$ & 0,22 \\
\hline Privada & 75 & $10-19$ & 16,0 & $15,1(2,3)$ & \\
\hline \multicolumn{6}{|l|}{ Categoria profissional } \\
\hline Enfermeiros & 20 & $11-18$ & 16,0 & $15,9(1,9)$ & 0,01 \\
\hline Não enfermeiros & 166 & $6-19$ & 15,0 & $14,8(2,3)$ & \\
\hline \multicolumn{6}{|l|}{ Carga horária semanal } \\
\hline Até 40 horas & 72 & $6-19$ & 15,0 & $14,5(2,7)$ & 0,96 \\
\hline Acima de 40 horas & 129 & $6-19$ & 15,0 & $14,9(2,2)$ & \\
\hline \multicolumn{6}{|c|}{ Outro vínculo empregatício } \\
\hline Sim & 43 & $6-18$ & 15,0 & $14,3(2,4)$ & 0,12 \\
\hline Não & 143 & $7-19$ & 15,0 & $14,9(2,4)$ & \\
\hline
\end{tabular}

* Desvio Padrão, ${ }^{\dagger}$ Mann - Whitney

Segundo os resultados apresentados na Tabela 2 podemos constatar que os enfermeiros apresentaram valor médio de demanda maior do que os demais profissionais da enfermagem, respectivamente, 15,9 e 14,8. Valores diferentes também foram observados entre os trabalhadores das instituições privadas (média de 15,1) quando comparados com os das instituições públicas/ filantrópicas (média de 14,6). Entretanto, apenas com relação à categoria profissional a diferença entre os grupos mostrou-se estatisticamente significante $(p=0,01)$.

Tabela 3 - Estatística descritiva da dimensão controle segundo a associação com as variáveis profissionais dos trabalhadores de enfermagem. Londrina-PR, 2007

\begin{tabular}{|c|c|c|c|c|c|}
\hline \multirow[t]{2}{*}{ Variáveis } & \multicolumn{5}{|c|}{ Controle } \\
\hline & $\mathrm{n}$ & Amplitude & Mediana & Média $\left(D^{*}\right)$ & $\mathrm{p} \dagger$ \\
\hline \multicolumn{6}{|l|}{ Tipo de instituição } \\
\hline Pública/filantrópica & 113 & $7-23$ & 17,0 & $16,9(2,4)$ & 0,01 \\
\hline Privada & 71 & $9-20$ & 16,0 & $16,0(2,2)$ & \\
\hline \multicolumn{6}{|l|}{ Categoria profissional } \\
\hline Enfermeiros & 21 & $14-21$ & 19,0 & $18,7(3,0)$ & 0,00 \\
\hline Não enfermeiros & 163 & $7-23$ & 16,0 & $16,3(2,3)$ & \\
\hline \multicolumn{6}{|l|}{ Carga horária semanal } \\
\hline Até 40 horas & 64 & $11-23$ & 17,5 & $17,3(2,2)$ & 0,00 \\
\hline Acima de 40 horas & 120 & $7-22$ & 16,0 & $16,2(2,4)$ & \\
\hline \multicolumn{6}{|c|}{ Outro vínculo empregatício } \\
\hline Sim & 44 & $7-22$ & 16.5 & $16.4(2.5)$ & 0.68 \\
\hline Não & 140 & $9-23$ & 17,0 & $16,6(2,3)$ & \\
\hline
\end{tabular}

* Desvio Padrão, ${ }^{\dagger}$ Teste $\mathrm{t}$ 
No que se refere aos resultados da dimensão controle, apresentados na Tabela 3, observamos diferenças estatisticamente significantes nas medidas dessa dimensão quando comparamos os profissionais segundo o tipo de instituição $(p=0,01)$, categoria profissional $(\mathrm{p}=0,00)$ e carga horária semanal $(p=0,01)$. Os profissionais que apresentaram melhor valor para a medida da dimensão controle foram os que atuavam em instituições públicas/ filantrópicas, da categoria enfermeiros e aqueles com menor carga horária semanal.

\section{DISCUSSÃO}

Como observado no presente estudo, trabalhadores de enfermagem constituem uma categoria essencialmente feminina, como comprovam outras pesquisas realizadas no Brasil. Dados publicados pelo Ministério do Trabalho e Emprego indicam que as mulheres representam $73 \%$ dos empregos formais da área da saúde e embora no mercado de trabalho brasileiro, a participação feminina tenha se intensificado a partir da década de 70, no trabalho de enfermagem, a predominância de mulheres essa sempre foi e continua presente. ${ }^{15}$

Estudo sobre as questões de gênero, características psicossociais do trabalho e o estado de saúde mostrou que as mulheres apresentam características mais negativas do que os homens, em especial a falta de oportunidade de crescimento no ambiente de trabalho, ${ }^{15}$ informação relevante para a enfermagem, uma vez que a profissão é predominantemente constituída por profissionais do sexo feminino.

O pressuposto de Karasek estabelece que o trabalho realizado em condições de baixo controle e alta demanda é nocivo à saúde dos trabalhadores, pois são considerados como trabalho de alta exigência. $O$ estudo entre professores e trabalhadores de enfermagem da Bahia corroborou o pressuposto do modelo demanda-controle, cujo resultado revelou que profissionais com trabalho de alta exigência apresentam elevada prevalência de Distúrbios Psíquicos Menores. ${ }^{12,16}$

Neste estudo, encontramos 56,1\% dos trabalhadores na categoria de exposição intermediária ao estresse ocupacional, porém devemos considerar que estes profissionais podem em outro momento perceber maior demanda e menor controle em seu ambiente de trabalho, tornando-se um grupo predominantemente de alta exposição ao estresse no trabalho e com maior risco para o adoecimento. Destacamos que em nosso estudo, 26,3\% dos par- ticipantes consideravam-se altamente expostos ao estresse, frequência considerada preocupante.

Na maioria dos hospitais o trabalho da enfermagem tem sido apontado como altamente estressante. $\mathrm{O}$ estresse do enfermeiro pode se justificar pela alta responsabilidade e pela baixa autonomia, as quais refletem situações com vários pontos de tensão, determinantes do estresse.

O fato da maioria dos trabalhadores do nosso estudo se encontrar na categoria de exposição intermediária ao estresse ocupacional pode ser justificado, pois estudos de corte transversal como o nosso, proporcionam apenas uma imagem instantânea da variável que se pretende estudar, havendo a necessidade de outros estudos com delineamentos distintos que possam avaliar o estresse ocupacional entre os profissionais de enfermagem no decorrer do tempo.

Tipo de instituição em que atuam os profissionais de enfermagem, categoria profissional, carga horária de trabalho e a presença de duplo vínculo empregatício são fatores importantes na vida dos trabalhadores, podendo estar associados à percepção do estresse ocupacional e por isso foram incluídos neste estudo.

Alguns autores consideram que o principal fator gerador de estresse no meio ambiente de trabalho decorre dos aspectos da organização, administração e sistema de trabalho e da qualidade das relações humanas. ${ }^{4}$ Esse clima torna o trabalho dos enfermeiros complexo e envolve uma grande tensão emocional, desgaste físico e psíquico o que contribui para desencadear estresse. Se analisarmos o resultado apresentado pelos enfermeiros deste estudo, de acordo com o modelo de estresse ocupacional estudado, eles encontram-se no quadrante de trabalhadores ativos, classificados como grupo de exposição intermediária ao estresse ocupacional. Esse aspecto pode ser justificado, pois o grau de controle pode indicar uma medida de autonomia, de liberdade para o uso de habilidades, compensando os efeitos negativos provenientes das altas demandas psicológicas.

Uma pesquisa entre enfermeiros envolvidos nas questões gerenciais, demonstrou que esses profissionais apresentaram seis vezes mais chances de apresentar altos níveis de estresse quando comparados a outros enfermeiros que não atuavam nessa área. ${ }^{7}$

Em estudo entre trabalhadoras de enfermagem foram constatadas associações importantes e estatisticamente significantes entre maiores cargas de demanda e categoria profissional e entre carga 
horária e alta demanda, ${ }^{10}$ divergindo do nosso resultado cuja associação não foi estatisticamente significante $(p=0,96)$. Na referida pesquisa, o baixo controle esteve relacionado com o número de horas trabalhadas e a ocupação. Resultado semelhante foi descrito em nosso estudo, onde encontramos associação estatisticamente significante entre a dimensão controle e essas variáveis.

Os enfermeiros apresentaram maior média para as dimensões demanda e controle quando comparados aos profissionais não enfermeiros, 0 que pode ser justificado pelo fato dos enfermeiros serem responsáveis pela administração e gestão de pessoal, pelo gerenciamento da assistência de enfermagem e responsabilizarem-se pelo gerenciamento dos conflitos e insatisfações e ainda encontrarem-se em nível superior, na hierarquia institucional, aos demais trabalhadores da enfermagem.

O nível de pressão exercido pela organização do trabalho, a exigência de maior produtividade, associada à redução contínua do contingente de trabalhadores, à pressão do tempo e ao aumento da complexidade das tarefas, além de expectativas irrealizáveis e as relações de trabalho tensas e precárias, podem gerar tensão, fadiga e esgotamento profissional, constituindo-se em fatores responsáveis por situações de estresse relacionado com o trabalho.

Estudos internacionais, realizados a partir da década de 90 tem dado importância à satisfação dos enfermeiros no trabalho (job satisfaction) e à permanência destes no emprego (retention), uma vez que algumas investigações têm apontado este elemento como um determinante importante para a garantia da qualidade da assistência prestada, redução de gastos hospitalares e diminuição de rotatividade de pessoal de enfermagem. ${ }^{17-18}$

Além de contribuir para o estresse ocupacional, a ausência de controle sobre o próprio trabalho frequentemente contribui para o aumento de sentimento de insatisfação profissional, podendo interferir na Qualidade de Vida no Trabalho (QVT) dos profissionais.

Concluímos neste estudo que o profissional de enfermagem do BC necessita de grande atenção no que diz respeito à sua saúde, uma vez que apenas $17,6 \%$ dos participantes não se consideravam estressados, ou seja, $82,4 \%$ encontravam-se estressados quer seja na categoria de exposição intermediária ou de alta exposição ao estresse no trabalho.

Os resultados deste estudo contribuem também para o avanço do conhecimento multidisciplinar da Saúde do Trabalhador e fornecem subsídios á área da Enfermagem, para o planejamento de medidas preventivas ao estresse ocupacional e para a promoção da QVT.

\section{REFERÊNCIAS}

1. Rossi AM. Estressores ocupacionais e diferenças de gênero. In: Rossi AM, Perrewé PL, Sauter SL. Stress e qualidade de vida no trabalho: perspectivas atuais da saúde ocupacional. São Paulo (SP): Atlas; 2005. p.9-18.

2. Limongi-França AC, Rodrigues AL. Stress e trabalho: uma abordagem psicossomática. $4^{\mathrm{a}}$ ed. São Paulo (SP): Atlas; 2005.

3. Murofuse, NT, Abranches, SS; Napoleão, AA. Reflexões sobre estresse e Burnout e a relação com a enfermagem. Rev Latino-am Enfermagem. 2005 Mar-Abr; 13(2):255-61.

4. Costa JRA, Lima JV, Almeida PC. Stress no trabalho do enfermeiro. Rev Esc Enferm USP. 2003 Set; 7(3):63-71.

5. Karasek RA, Theorell T. Healthy work. New York (US): Basic Books; 1990.

6. Baker D, Karasek RA. Stress. In: Levy BS, Wegman DH. Occupational health: recognizing and preventing work-related disease and injury. $4^{\mathrm{a}}$ ed. Philadelphia (US): Lippincott Williams \& Wilkins; 2000.

7. Lindholm M. Working conditions, psychosocial resources and work stress in nurses and physicians in chief managers' positions. J Nurs Manage. 2006 Mar; 14(2):300-9.

8. Aquino JM. Estressores no trabalho das enfermeiras de centro cirúrgico: conseqüências profissionais e pessoais [tese]. Ribeirão Preto (SP): Universidade de São Paulo. Escola de Enfermagem de Ribeirão Preto; 2005.

9. Bianchi ERF. Comparação do nível de estresse do enfermeiro de centro cirúrgico e de outras unidades fechadas. Rev SOBECC. 2000 Out-Dez; 5(4):28-30.

10. Alves MGM. Pressão no trabalho: estresse no trabalho e hipertensão arterial em mulheres no Estudo Pró-Saúde [tese]. Rio de Janeiro (RJ): Escola Nacional de Saúde Pública; 2004.

11. Theorell T, Perski A, Akerstedt T, Sigala F, AhlbergHultén G, Svensson J, et al. Changes in job strain in relation to changes in psychological state. Scand J Work Environ Health. 1988 Jun; 14(3):189-96.

12. Araújo TM, Aquino E, Menezes G, Santos CO, Aguiar L. Aspectos psicossociais do trabalho e distúrbios psíquicos entre trabalhadores de enfermagem. Rev Saúde Pública. 2003 Ago; 37(4):424-33.

13. Cohen J, Cohen P. Applied multiple regression/ correlation for the behavioral sciences. $2^{\mathrm{a}}$ ed. New Jersey (US): Hillsdale; 1983.

14. Urbina S. Fundamentos em Fidedignidade. In: Urbina S. Fundamentos da Testagem Psicológica. Porto Alegre (RS): Artmed; 2007. p.121-53. 
15. Girardi SM, Carvalho CL. Configurações do mercado de trabalho dos assalariados em saúde no Brasil [on line]. Organização Pan-Americana de saúde; 2003 [acesso em 2008 Jun]. Disponível em: http:/ / www. opas.org.br/rh/admin/documentos/mtlast.PDF

16. Reis EJFB, Araújo TM, Carvalho FM, Barbalho L, Silva MO. Docência e exaustão emocional. Educ Soc. 2006 Jan-Abr; 27(94):229-53.
17. Lu H, While AE, Barriball KL. Job satisfaction among nurses: a literature review. Int J Nurs Study. 2005 Fev; 42(2):211-7.

18. Mayyan MT. Nurse job satisfaction and retention: comparing public to private hospitals in Jordan. J Nurs Manage. 2005 Jan; 13(1):40-50. 